\title{
A New Method of Segmentation of Compact-Appearing, Transitional and Trabecular Compartments and Quantification of Cortical Porosity from High Resolution Peripheral Quantitative Computed Tomographic Images
}

Running title: Segmentation by Non-Threshold Based Image Analysis

$$
\begin{gathered}
\text { R Zebaze }^{1,2} \\
\text { A Ghasem Zadeh }^{1,2}
\end{gathered}
$$

A Mbala ${ }^{2}$

E Seeman $^{1,2}$

${ }^{1}$ Dept Endocrinology and Medicine, Austin Health, University of Melbourne, Melbourne, Australia and ${ }^{2}$ StraxCorp PTY LTD

Dr R Zebaze. Dept Endocrinology, level 2, Centaur Wing, Repatriation Campus, Austin Health, Heidelberg 3081, Melbourne, Victoria, Australia. Tel 61394965489. Fax 6139496 3365. zebaze@unimelb.edu.au 


\begin{abstract}
A transitional or cortico-trabecular junctional zone exists at any location composed of both cortical and trabecular bone such as the metaphyses of tubular bones and short bones like the femoral neck. The transitional zone comprises the inner cortex adjacent to the medullary canal and trabeculae abutting against the cortex contiguous with the endocortical surface. This is a site of vigorous remodeling. Intracortical remodeling cavitates the inner cortex expanding this transitional zone at the price of compact-appearing cortex so that it contains porosity, cortical fragments that resemble trabeculae, and trabeculae abutting the eroding cortex. The porosity of the transitional zone reduces bone strength exponentially and is a quantifiable 'fingerprint' of structural deterioration.
\end{abstract}

A new automated method of segmentation of bone from background and bone into its compact-appearing cortex, transitional zone, and trabecular compartment is described, with a new approach to quantification of cortical porosity. Segmentation is achieved by automatically selecting attenuation profile curves perpendicular to the periosteal surface. Local bone edges are identified as the beginning and the end of the rising and falling S-shaped portions of the curve enabling the delineation of the compartments. Analysing 3600 consecutive overlapping profiles around the perimeter of each cross-sectional slice segments the compartments.

Porosity is quantified as the average void volume fraction of all voxels within each compartment. To assess accuracy at the distal radius and tibia, $\mu \mathrm{CT}$ images of cadaveric specimens imaged at $19 \mu \mathrm{m}$ voxel size served as the gold standard. To assess accuracy at the proximal femur, scanning electron microscopy (SEM) images of specimens collected at $2.5 \mu \mathrm{m}$ resolution served as the gold standard.

Agreement between HRpQCT and the gold standards for segmentation and quantification of porosity at the distal radius and tibia ranged from $\mathrm{R}^{2}=0.87$ to 0.99 , and for the proximal femur ranged from 0.93 to 0.99 . The precision error in vivo for segmentation and quantification of porosity in HRpQCT images at the distal radius, given by the root mean square error of the coefficient of variation, ranged from $0.54 \%$ for porosity of the transitional zone to $3.98 \%$ for area of the compact-appearing cortex.

Segmentation of the transitional zone minimizes errors in apportioning cortical fragments and cortical porosity to the medullary compartment and so is likely to allow accurate assessment of fracture risk and the morphological effects of growth, ageing, diseases and therapies.

Key Words Cortical Bone, Curve Profile Analysis, Edge Finding, Image Analysis, Microstructure, Non-Threshold Based Segmentation, Porosity, Transitional Zone. 


\section{Introduction}

Eighty percent of the skeleton's mineralized bone matrix is cortical, $20 \%$ is trabecular. Of all age-related appendicular bone loss, $\sim 70 \%$ is cortical and is the result of intracortical remodeling initiated upon Haversian canals traversing the cortex [1]. The inner cortex adjacent to the medullary canal and the trabeculae abutting against the endocortical surface form the cortico-trabecular junctional region or transitional zone $[2,3]$.

Intracortical remodeling is particularly vigorous in this junctional region between cortical and trabecular compartments [4,5], and of all the cortical bone lost during aging, about half arises from intracortical remodeling within this narrow transitional zone [1]. The inner compact-appearing cortical region cavitates and leaves fragments, expanding the transitional zone radially at the expense of the progressively more porous, but still compact-appearing, cortex which thins from 'within' and contains more porosity $[1,6]$.

Measurement of the morphology of the cortical bone and particularly the transitional zone is important because it is the source of most bone loss [1]. The resulting porosity reduces bone strength exponentially [7], and is a quantifiable 'fingerprint' of structural deterioration which is likely to predict fracture risk and may be used as a marker of responsiveness to therapy [8]. Many methods are used to segment bone. However, none segment the transitional zone in HRpQCT images. We report an accurate and reproducible method of segmenting bone from background, segmenting bone into its compact-appearing, transitional zone, and trabecular compartments, and quantifying porosity in vivo.

\section{Materials and Methods}

\section{Segmentation of Compact-Appearing Cortex, Transitional Zone, and the Trabecular Compartment}

Ex vivo and in vivo images were processed using StrAx1.0, a custom software using a segmentation algorithm fully described in the patent [9]. Image processing begins by identifying a point ' $\mathrm{C}$ ' within the medullary cavity which may be the centroid but this is not essential (fig 1). A rectangular region of interest (ROI) referred to as an 'Arm' starts at ' $\mathrm{C}$ ' and extends to the edge of the entire image (Po). To segment, the algorithm operates from ' $\mathrm{C}$ '. The Arm is selected so that its length is perpendicular to the bone surface. The Arm width (AW) is selected to be narrow enough to ensure that the periosteal edge of the bone within the Arm is locally linear but contains two or more rows of voxels to avoid discontinuities due to noise or pores resulting in irregularities of bone boundaries after segmentation. $\mathrm{AW}=\mathrm{R}_{\mathrm{i}}^{*} \tan (\operatorname{acos}(\Delta))$ and is automatically quantified as a function of the distance between ' $\mathrm{C}$ ' and the nearest periosteal surface $\left(\mathrm{R}_{\mathrm{i}}\right)$. A delta $(\Delta)$ value of 0.98 is used because it is close to 1 so that the local portion of the periosteal surface is almost linear. 
The attenuation profile curve within the Arm is produced by the background, compact-appearing cortex, transitional zone and trabecular bone. At the background/bone edge, the attenuation profile curve forms an $\mathrm{S}$-shape. A point $\mathrm{P}_{1}$ is selected from which the beginning, ' $\mathrm{K}$ ', of the sharp rise of the curve, is identified and corresponds to the beginning of the transition from background to mineralized bone (see legend of figure 1 for details). Point ' $\mathrm{K}$ ' is the intersection between the circle of smallest radius originating from $\mathrm{P}_{1}$ and the curve. The $x, y$ coordinates (position along the Arm, attenuation) of ' $\mathrm{K}$ ' are calculated. From ' $\mathrm{K}$ ', a new referent $\mathrm{P}_{2}$ is calculated from which the circle of smallest radius intersecting the curve identifies the end of the background/bone edge, ' $\mathrm{M}$ '; the beginning of the compactappearing cortex. ' $M$ ' is identified after eliminating voxels between ' $K$ ' and ' $M$ ' as they may contain both the void volume of the adjacent background and the mineralized bone matrix of the cortex. Inclusion of the background void may overestimate porosity during quantification of cortical porosity (partial volume effects, see Discussion).

After finding the external bone edge within the Arm, the algorithm analyzes the inverted $\mathrm{S}$ shape of the remainder of the curve between $\mathrm{P}_{1}$ and ' $\mathrm{C}$ ' capturing the cortex, the transition from the cortex to trabecular bone, and trabecular bone in the medullary cavity (fig 1). The algorithm starts again from referent $\mathrm{P}_{1}$. It first identifies the end of the sharp descent of the $\mathrm{S}$ curve, ' $\mathrm{O}$ ', which corresponds to the beginning of trabecular bone. From ' $\mathrm{O}$ ', a new referent $\mathrm{P}_{3}$ is calculated from which ' $\mathrm{N}$ ', end of the compact-appearing cortex and beginning of the transitional zone is identified. The descent of the inverted $\mathrm{S}$ shaped curve from ' $\mathrm{N}$ ' to ' $\mathrm{O}$ ' is the transitional zone (fig 2). The $x, y$ coordinates identifying these plateaus, and the transition between them, are automatically and objectively identified by the algorithm.

After analyzing the first ROI, the Arm sweeps in steps of 0.1 degrees (3600 ROIs per cross section) analyzing consecutive and overlapping Arms (ROIs) until a rotation of 360 degrees is completed and the entire cross-sectional slice has been assessed. For HRpQCT images, the analysis is restricted to the 40 most proximal slices because the 70 distal slices often have very thin cortices. These cortices can be segmented but voxels containing them may also contain background void, trabecular bone or medullary void, and so they are unsuitable for unambiguous quantification of cortical porosity.

Rotation of the Arm every 0.1 degrees ensures that every voxel is reanalyzed many times. For an HRpQCT image of the distal radius or tibia, a voxel may reappear in several hundred overlapping Arms. Ultimately, each voxel location is attributed to the compartment in which it has appeared the maximum number of times. This multicategorization of voxel locations minimizes segmentation errors and is an essential feature of this 'edge finder' algorithm. The area of each compartment is $A_{r e a}=N^{*} R$ where $\mathrm{N}$ is the number of voxels in the compartment and $\mathrm{R}$ is the nominal voxel size.

For clarity of description, segmentation is described in the context of a 2-D cross section with the 'Arm' rotating in the $x-y$ plane. However, the 'Arm' also rotates in the $\mathrm{x}-\mathrm{z}$ direction to achieve $3 \mathrm{D}$ segmentation. 


\section{Quantification of Porosity}

Quantification of porosity in vivo is challenging because the median size of Haversian canals is $\sim 50 \mu \mathrm{m}[10,11]$. A significant proportion of voxels overlying cortical bone are composed of varying proportions of void volume and mineralized bone matrix volume. Thus, porosity is quantified voxel by voxel. The proportion of the voxel volume occupied by mineralized bone matrix volume is its level of fullness (LOF). LOF is estimated by knowing the attenuation of a voxel that contains no mineralized bone (having the attenuation of background) and the attenuation of a voxel that contains only mineralized bone. If $\mathrm{I}_{\mathrm{i}}$ is the attenuation of voxel ' $\mathrm{i}$ ', $\mathrm{P}$ is background attenuation, $\mathrm{B}$ is the attenuation produced by a voxel containing only fully mineralized bone then the $\mathrm{LOF}_{i}(\%)$ of voxel ' $\mathrm{i}$ ' $=\left(\mathrm{I}_{\mathrm{i}}-\mathrm{P}\right) /(\mathrm{B}-\mathrm{P})$.

As LOF is a relative measure normalized to $\mathrm{P}$ and $\mathrm{B}$, it is independent of the image source, e.g., scanning electron microscopy or scanner type. . When analysing images of high resolution such as an SEM image, the attenuation of $\mathrm{P}$ and $\mathrm{B}$ are retrieved automatically by an algorithm described in Appendix I. However, for HRpQCT images, $\mathrm{P}$ and $\mathrm{B}$ are obtained by scanning phantoms of different hydroxyapatite concentrations. Fully mineralized bone has an attenuation equivalent to that produced by hydroxyapatite (HA) of $1200 \mathrm{mgHA} / \mathrm{cc}$ [12]. StrAx1.0 uses the values of P and B to automatically estimate the LOF of individual voxels.

The void content or level of emptiness (LOE) of each voxel (i.e., porosity) is $100-$ LOF (\%). The porosity of the entire compartment (Po) is the average of all LOEs of all voxels $i\left(\mathrm{LOE}_{i}\right)$ where $n=$ total number of voxels within the compartment.

$$
P o=\sum_{i=1}^{i=n} L O E_{i} / \mathrm{n}
$$

Voxels may contain bone matrix at different stages of completeness of secondary mineralization which may produce errors in the quantification of porosity. The potential errors are addressed in the Appendix 2.

\section{Imaging Bone Specimens Ex Vivo and Bone Regions In Vivo}

\section{Accuracy at the distal radius and distal tibia}

Five distal radii, four distal tibia, and four specimens of the right femur were obtained from Caucasian cadavers. To assess the accuracy of StrAx1.0 in segmenting compartments and quantifying porosity, the radii and tibia were scanned using VivaCT 40 at $19 \mu \mathrm{m}$ voxel size as the 'gold' standard $(70 \mathrm{kVp}, 114 \mathrm{~mA}, 381 \mathrm{~ms}$ integration time, Scanco Medical, Bruttisellen, Switzerland) [13]. A point $9.02 \mathrm{~mm}$ proximal to the radius endplate was identified and 475 slices proximal to this point forms the ROI for $\mu \mathrm{CT}$ analysis. In these $\mu \mathrm{CT}$ images, porosity was quantified as the fraction of void voxels in the compartment. The same ROI was scanned using HRpQCT ( $82 \mu \mathrm{m}$ voxel size); 110 slices corresponding to the 475 slices were chosen. For the tibia, the ROI chosen was the $22.5 \mathrm{~mm}$ distance from the endplate as the first slice. In each site, only the 40 most proximal slices were quantified with HRpQCT for reasons discussed above, corresponding to 172 slices measured with $\mu \mathrm{CT}$. 


\section{Accuracy at the proximal femur (subtrochanteric region)}

For femur samples, an SEM image of the first (top) slice was collected (resolution 2.5 $\mu \mathrm{m})$ and served as the 'gold' standard. This SEM image is a map of the surface of this first cross section. These SEM images were first processed manually by experts (RZ \& AGZ) using Image J (NIH, USA), segmenting the compact-appearing cortex, transitional zone, and trabecular compartment based on histomorphometric features. The HRpQCT image (voxel size $82 \mu \mathrm{m}$ ) of the same top slice was obtained. Hence, the first cross sectional slice obtained using HRpQCT is similar to the SEM although the HRpQCT slice was $\sim 82 \mu \mathrm{m}$ thick whereas the SEM has no thickness. The accuracy of StrAx1.0 was assessed by comparing its ability to segment the compartments and quantify porosity in the SEM images, and in the corresponding HRpQCT images.

\section{Agreement between StrAx1.0 and manual contouring of in vivo HRpQCT images}

To test the agreement between $\operatorname{StrAx} 1.0$ and manual contouring of the compactappearing cortex of HRpQCT images, 23 distal radii and 15 distal tibia images were selected randomly from the study population. Compact-appearing cortex and the medullary canal were manually segmented by experts (RZ \& AGZ). As the transitional zone is a thin irregular layer, it was not possible to manually delineate this compartment at this resolution.

\section{Agreement between StrAx1.0 and Scanco HRpQCT in vivo images}

To test the agreement between StrAx1.0 and Scanco, we analysed the same ROI in in vivo HR-pQCT images of distal radius and tibia in 90 females aged 20-99 years without illnesses or exposure to therapy known to affect bone. For both methods, analyses were restricted to the 40 proximal most slices. Quantification of porosity using Scanco was done as previously reported [14-16]. All participants gave written informed consent. The study was approved by Austin Health Ethics Committee.

Precision Seven women had four measurements each. Images were then visually co-registered by an expert (AGZ) and analyzed using StrAx1.0 to test the effect of acquisition plus repositioning, co-registration, and processing on segmentation and quantification of porosity.

\section{Statistical Analysis}

Data were analyzed using SPSS 16.0 for Mac and expressed in absolute terms, percentage differences (with SEM), standard deviations (SD), and coefficients of variation ( $\mathrm{SD} / \mathrm{mean})$. To validate $\mathrm{StrAx} 1.0$ segmentation and quantification of porosity, linear regression was used to determine the $\mathrm{R}^{2}$ between the gold standard and StrAx1.0 measurements. The root mean square errors (RMSE) between the StrAx1.0 measurements and the gold standard were quantified. Bland-Altman plots were used to assess differences between any two measurement methods. To measure the overlap rate between two segmentations, we quantified the Dice ratio (DR) or the similarity index. For two segmented regions $A$ and $B$, the $D R=2|A \cap B| /(|A|+|B|)$, which ranges from 0 (worst) to 1 (best) agreement between the region segmented by 
StrAx1.0 and the gold standard [17]. The precision error was calculated as the RMS of the coefficients of variation $(\mathrm{CV} \%)$. Significance levels $(\mathrm{p}<0.05)$ were two-tailed.

\section{Results \\ Accuracy of distal radius and distal tibia image analyses}

For specimens of the distal radius, total cross sectional area (T-CSA), the compactappearing cortex, transitional, and medullary areas measured using HRpQCT predicted the gold standard $\mu \mathrm{CT}$ measurements $\left(\mathrm{R}^{2}\right.$ ranging from 0.95 to 0.99 ; all $\mathrm{P}<$ 0.05). The respective RMSEs were 3.93, 0.83, 0.74, and $4.13 \mathrm{~mm}^{2}$. Bland-Altman plots showed that the compact-appearing cortical areas were underestimated by 7.15 $\mathrm{mm}^{2}$ and transitional zone areas were overestimated by $4.73 \mathrm{~mm}^{2}$ in HRpQCT measurements (fig 3). Compact-appearing cortex and transitional zone porosities quantified from HRpQCT images predicted the gold standard from $\mu C T$ images $\left(\mathrm{R}^{2}=\right.$ 0.87 and 0.98 respectively; all $\mathrm{P}<0.05$ ). Their respective RMSEs were $4.08 \%$ and $0.79 \%$ (fig 4). Bland-Altman plots showed no under- or over-estimation of the compact-appearing cortical porosity. Transitional zone porosity was underestimated by $4.98 \%$ (fig 4). Results were similar for the distal tibia (see legends figs 5 and 6 ).

\section{Accuracy of proximal femur image analysis}

T-CSA, compact-appearing cortex, transitional and medullary areas on the SEM image measured manually were $635.91 \pm 20.69,323.18 \pm 32.27,74.51 \pm 14.12$, and $237.33 \pm 19.53 \mathrm{~mm}^{2}$ respectively. The respective areas on the same SEM image measured by StrAx1.0 were $627.17 \pm 21.5,316.22 \pm 35.2,66.98 \pm 9.79$, and $241.58 \pm$ $22.29 \mathrm{~mm}^{2}$. The respective areas of the HRpQCT image corresponding to the SEM image measured by StrAx1.0 were $613.79 \pm 35.61,330.08 \pm 33.56,75.39 \pm 12.96$, and $207.15 \pm 26.75 \mathrm{~mm}^{2}$. Areas measured manually correlated with areas measured on SEM images, and with corresponding areas on HRpQCT images, $\mathrm{R}^{2}$ ranged from 0.93 to 0.99 . RMSE ranged from 3.07 to $25.96 \mathrm{~mm}^{2}$ (table 1 ).

Dice ratios for T-CSA, compact-appearing cortex, transitional zone and medullary areas segmented manually and by StrAx1.0 were $0.99 \pm 0.01,0.98 \pm 0.08,0.98 \pm$ $0.01,0.93 \pm 0.01$, and $0.98 \pm 0.05$ respectively. Porosity of the compact-appearing cortex plus transitional zone was $18.78 \pm 5.27 \%$ measured manually on the SEM image, $18.51 \pm 5.35 \%$ measured by StrAx 1.0 on the same SEM image, and $22.23 \pm$ $6.54 \%$ measured by StrAx1.0 of the HRpQCT image of the corresponding region. Porosity measured manually correlated with porosity measured on SEM image, and on the corresponding HRpQCT image with $\mathrm{R}^{2}=0.97$ for both. RMSE were $1.85 \%$ and $2.3 \%$ for quantification of porosity on the SEM and HRpQCT images respectively (table 1).

\section{Agreement between StrAx1.0 and manual contouring of in vivo HRpQCT images}

Correlations between T-CSA and area of the compact-appearing cortex measured manually and by StrAx1.0 ranged from an $\mathrm{r}^{2}$ of 0.94 to 0.99 depending on the trait and site measured (see figure 7 and legend). Bland-Altman plots show that there was agreement between the methods (fig 7). 


\section{Agreement between StrAx1.0 and Scanco HRpQCT in vivo images}

T-CSA measured by Scanco and StrAx1.0 from HRpQCT images of distal radius in vivo correlated $\left(\mathrm{R}^{2}=0.99\right)$. StrAx 1.0 measured a smaller T-CSA $\left(205.0 \pm 3.4 \mathrm{~mm}^{2}\right)$ than Scanco $\left(213.6 \pm 3.3 \mathrm{~mm}^{2}, \mathrm{p}=0.07\right)$ (fig 8). The compact-appearing cortical area defined by StrAx1.0 and the cortical area defined by Scanco correlated; $R^{2}=0.81$, but the area measured by StrAx 1.0 was smaller than measured by Scanco $(41.42 \pm 0.78$ vs. $\left.58.35 \pm 1.14 \mathrm{~mm}^{2}, \mathrm{p}<0.05\right)$; the larger the cortex, the greater the differences $\left(\mathrm{R}^{2}=\right.$ $0.44, \mathrm{p}<0.05)$. Medullary area measured by $\operatorname{StrAx} 1.0$ was smaller than measured by Scanco $\left(123.4 \pm 2.74\right.$ vs. $\left.152.4 \pm 3.34 \mathrm{~mm}^{2}, \mathrm{p}<0.05\right)$. The Bland-Altman plot showed the larger the 'true' medullary area (i.e., mean of both methods), the greater the difference between them $\left(\mathrm{R}^{2}=0.81, \mathrm{p}<0.05\right)$. Nevertheless, medullary area measured by each method correlated $\left(r^{2}=99.1\right)$ (fig 9, Appendix fig 1).

Precision The precision error for segmentation and porosity quantification expressed as RMSCV ranged from $0.54 \%$ to $3.98 \%$ (table 2 ).

\section{Discussion}

We report a new automated method of segmentation of high resolution images acquired using HRpQCT and SEM. The method accurately and reproducibly separates bone from background, separates bone into its cortical, transitional and trabecular compartments, and quantifies cortical porosity below (as well as above) $100 \mu \mathrm{m}$, the size of most pores in cortical bone $[10,11]$

To segment, the algorithm uses a rectangular ROI or 'Arm'. At the background/bone edge there is a sharp rise in the attenuation profile due to the increase in the amount of mineralized bone matrix volume within voxels. The initial part of this rise identifies the end of the background, the end of this rise identifies the beginning of the compactappearing cortex. Between these two points are composite voxels containing void from the background and mineralized bone matrix of the cortex. These voxels are excluded when porosity is calculated because the inclusion of voids due to background in these edge voxels overestimates porosity. The voxels are retained and quantified if the question concerns periosteal apposition (see Appendix fig 2).

Segmentation into a three compartments by StrAx1.0 is driven by the biology of bone. The measurement of the microarchitecture of the transitional zone is essential if the structural basis of the attainment of peak bone strength and its loss with age is to be understood. A transitional or cortico-trabecular junctional zone exists at any location composed of both cortical and trabecular bone such as the metaphyses of tubular bones and short bones like the femoral neck [2-5]. The transitional zone is assembled as growth plate trabeculae lengthen and coalesce forming the metaphyseal cortical bone [18]. Dissociation between accelerated longitudinal growth of the distal radial metaphysis and a relative delay in coalesce ('corticalization') of adjacent trabeculae as they emerge from the growth plate produces porosity in the transitional zone predisposing to fractures in childhood [19].

The transitional zone is also generated during advancing aging as accelerated intracortical and endocortical remodeling cause bone loss. Intracortical remodeling 
increases intracortical porosity, particularly in the inner cortex where pores enlarge focally, coalesce and cavitate thinning the cortex from within $[1,6]$. The compactappearing cortex is replaced by cortical fragments and pores so that it resembles the medullary cavity containing what appears to be 'trabeculae' $[1,6]$. The porosity and cortical fragments produced may be incorrectly apportioned to the trabecular (medullary) compartment rather than cortical compartment using noninvasive imaging or histomorphometry leading to an underestimate of the age-related rise in cortical porosity and an underestimate of the age-related decrease in trabecular density [1].

The term transitional zone is preferable to 'trabecularized' cortex. The transitional zone does not only contain cortical fragments, it also contains true trabeculae arising from the growth plate that buttress the endocortical surface conferring strength to the metaphysis, perhaps serving to minimize buckling of the cortex $[2,3]$. While cortical fragments have a similar appearance to trabeculae, they are thicker and have a chaotic architecture that may confer less strength than the cancellous architecture that is also eroding. Moreover, a three compartment model (cortical-transitional-trabecular) may minimize the inclusion of cortical fragments in the trabecular compartment avoiding the underestimate in age-related trabecular bone loss (see figure 9 and Appendix figure 1).

Many algorithms use attenuation profile curve analysis but they only segment bone into two, cortical and trabecular, compartments [20-24]. Mastmeyer et al. segment bone into cortical, subcortical, trabecular and many other regions of interest based on CT images of the hip and spine [25]. However, StrAx1.0 is currently the only method that delineates and separates the transitional zone from the compact-appearing cortex and the trabecular compartment in HRpQCT images. For this reason, StrAx1.0 also differs from the manufacturer (threshold-filter) and the dual threshold segmentation method [14-16, 26].

StrAx1.0 quantified a slightly but not significantly smaller distal radius total CSA than Scanco because StrAx1.0 nominated the end of the ascending portion of the S shaped section of the attenuation profile curve as the periosteal boundary excluding composite voxels at the junction between bone and background (see Appendix fig 2). StrAx1.0 also quantified a smaller medullary canal because the transitional zone is recognised. StrAx1.0 segmented the medullary volume occupied by trabecular bone and identified trabecular bone voxels as those with attenuation above background values. Individual trabeculae are not segmented so that trabecular thicknesses or connectivity are not measured at this time.

As compared to the gold standard, StrAx1.0 analysis of HRpQCT images overestimated of the size of the transitional zone at the distal radius and tibia because composite voxels at the junction of the compact-appearing cortex and the transitional zone are allocated to the transitional zone. At $82 \mu \mathrm{m}$, these composite voxels are larger than at $19 \mu \mathrm{m}$ so that a larger amount of the compact-appearing cortex is allocated to the transitional zone in HRpQCT images at the expense of the compactappearing cortex. This only modestly underestimated the compact-appearing cortical area and did not affect its porosity because it is a large compartment. In the transitional zone, a smaller area, this resolution-dependent phenomenon produces a modest overestimate of area $(<5 \%)$ and underestimated its porosity by $\sim 5 \%$ (because a small fraction of the less porous compact-appearing cortex is now included in the 
transitional zone). Nevertheless, segmenting a transitional zone is advantageous as edge voxels will otherwise be allocated to the cortical or trabecular compartment. Overestimation of the transitional zone was not observed at the proximal femur because the transitional zone is larger.

StrAx1.0 quantifies porosity below, as well as above, the voxel size of the imaging device. This is achieved by the algorithm because it averages the void volume fraction of all voxels within the compartment. This key feature accounts for the accuracy in calculating porosity. Intracortical porosity is 20 to $25 \%$ in the compactappearing cortex; $\sim 70 \%$ in the transitional zone and so that average porosity in the total cortex is 30 to $40 \%$. These values are 2 to 3 fold higher than reported by other imaging methods [27-29], but they accord with rigorous ex vivo assessment of porosity done by measuring the water content of bone [12,30-32]. Engelke et al. also report porosity values of $\sim 20 \%$ in HRpQCT images of the distal radius and distal tibia [33].

The void volume fraction (porosity) is a surrogate of bone water content because $90 \%$ of the water is in the Haversian-Volkmann canals, the rest is matrix bound [12, 30]. Bone water content of the compact-appearing cortex is rarely below $10 \%$, and averaging $\sim 20 \%$ when assessed by methods such as replacing bone water by deuterium oxide water [12,30-32]]. This canal system provides space for vascularization of bone. Goldenstein et al. and Melton et al. report average porosity values of $\sim 1 \%$ at the distal radius [27,28]. Porosities reported by Nishiyama et al., using the auto-segmentation are $5.1 \%$ in premenopausal to $13.6 \%$ in postmenopausal women at the distal radius [14]. Values of porosity below 5\% are difficult to reconcile with the experiments using deuterium oxide water and the need for pores in sufficient amounts to provide an effective vascular supply to bone cells. Values of $20.5 \%$ reported by Nishiyama et al. using autosegmentation are similar to those we report within the compact-appearing cortex at the distal tibia [14].

Errors in porosity may arise when there are coexisting differences in the degree of completeness of mineralization between regions. Newly remodelled bone matrix undergoes rapid primary mineralization reaching $\sim 80$ percent of peak matrix mineralization in days to weeks [34]. Thus, little of the differences in mineralization between regions is due to incomplete primary mineralization; the relative osteoid volume is less than $0.5 \%$ in cortical bone [35]. The heterogeneity of mineralization is largely due to the incompleteness of secondary mineralization which can take 1 to 3 years, if not more $[36,37]$.

Voxels containing bone matrix undergoing secondary mineralization have an attenuation value equal or greater than $80 \%$ of the maximum [38, also see appendix 2]. These voxels may be interpreted as containing a pore and so are excluded from quantification of porosity by StrAx1.0 (see Appendix 2). Attenuation values $<80 \%$ of the peak attenuation signals the presence of a pore; this level of attenuation is unlikely to be due to submaximally mineralized bone matrix alone.

Prolonged antiresorptive therapy may increase the degree of completeness of secondary mineralization which in turn increases attenuation values which may be interpreted to reflect a decrease in porosity. Exclusion of these voxels containing bone at different stages of secondary mineralization minimizes this potential 
confounding effect of mineralization. The potential error introduced by mineralization heterogeneity also applies to threshold based methods which may include composite voxels. Porosity will be underestimated because the voxel (with its void volume) will be disregarded as the total voxel attenuation exceeds that defining 'porosity'.

The method has several limitations. The size and shape of pores are not quantified and the location within the voxel is not known. A pore may be a stand-alone or be part of a large pore. Calculation of porosity is dependent on the local attenuation. In the case of CT images, this value may be altered in the vicinity of void spaces due to local inhomogeneity. However, the accuracy error in quantifying porosity introduced by this effect is small as the total porosity is the average of all values [39]. Furthermore, these inaccuracies are inherent to the image acquisition modality and do not apply to the analysis of SEM images by StrAx1.0. Osteomalacia may widen bone matrix mineralization heterogeneity beyond its physiological range. In this instance, the method may be unsuitable for quantification of porosity. We assume a voxel which is exclusively void is similar to that of background (i.e., muscle tissue). Pores containing fat may affect the accuracy of porosity calculation. However, any such effect is likely to be small as fat occupies less $0.5 \%$ of void spaces in the cortex [12].

Another limitation is the small number of ex vivo distal radius and distal tibia specimens used to assess the accuracy as compared to gold standard $\mu \mathrm{CT}$. However, we did test the agreement between manual segmentation and StrAx1.0 at the distal radius and tibia using a much larger sample size. Finally, the measurement of porosity by StrAx1.0 was compared to that of an earlier version of the autosegmentation method such as described by Burghardt et al. [14-16]. Comparison with more recent versions of the autosegmentation method will be the subject of future work.

In summary, studies of the effects of growth, ageing, disease, fracture risk assessment in individuals, and the effects of therapy on fracture risk are hindered, in part, by issues related to image processing and analysis. The method described here accurately and reproducibly (i) segments bone from background, (ii) segments bone into its compartments by finding the position of the bone edges and (iii) quantifies porosity by estimating the fraction of each voxel occupied by void space and averaging the porosities in all voxels. Segmentation of the transitional zone is likely to allow accurate assessment of the effects of growth, ageing, diseases and therapies by more correctly apportioning bone and bone loss to the cortical, transitional and trabecular compartments.

Acknowledgments This work was supported by a grant from the Australia National Health and Medical Research Council (Project Grants 566916 \& 628701). We thank the anonymous peer reviewers and the editor for their constructive criticisms, and Dr Y Peng of Straxcorp, for assistance in image generation. 


\section{References}

1. Zebaze RM, Ghasem-Zadeh A, Bohte A, Iuliano-Burns S, Mirams M, Price RI, Mackie EJ, Seeman E. Intracortical remodelling and porosity in the distal radius and post-mortems femurs of women: a cross sectional study. Lancet. 2010; 375:1729-36.

2. Keshawarz NM, Recker RR. Expansion of the medullary cavity at the expense of cortex in postmenopausal osteoporosis. Metab. Bone Dis. \& Rel. Res 1984;5: 223-8.

3- Palnitkar S, Rao D S, Nelson D, Parfitt AM. Effects of ethnicity and age or menopause on the remodeling and turnover of iliac bone: implications for mechanism of bone loss. J Bone Min Res. 1997;12: 498-508.

4. Brown JP, Delmas PD, Arlot M. Active bone turnover of the cortico-endosteal envelope in postmenopausal osteoporosis J Clin Endoc Metab. 1987; 64: 954-59

5. Foldes J, Parfitt AM, Shih M-S, Rao DS, Kleerekoper M. Structural and geometric changes in iliac bone: Relationship to normal aging and osteoporosis. J Bone Miner Res. 1991; 6:759-66.

6. Bell KL, Loveridge N, Power J, Garrahan N, Meggitt BF, Reeve J. Regional differences in cortical porosity in fractured femoral neck. Bone 1999;24:57-64.

7. Schaffler MB, Burr DB. Stiffness of compact bone: effects of porosity and density. J Biomechanics 1988;21:13-16.

8. Chiang CY, Zebaze RMD, Ghasem-Zadeh A, Iuliano-Burns S, Hardidge A, Seeman E. Teriparatide improves bone quality and healing of atypical femoral fractures associated with bisphosphonate therapy. Bone 2013; 52: 360-5

9. Zebaze R, GhasemZadeh A, Mbala A, and Seeman E. Method and system for image analysis of selected tissue structures. 2009

www.patentscope.wipo.int/search/en/WO2011029153.

10. Thompson DD. Age changes in bone mineralization, cortical thickness, and haversian canal area. Calcif Tissue Int. 1980;31:5-11.

11. Qiu S, Fyhrie PD, Palnitkar S, Rao DS, Histomorphometric assessment of Haversian canal and osteocyte lacunae in different-sized osteons in human rib. Anatomical Record Part A 2003; 272A:520-5

12. Muller KH, Trias A, Ray R. Bone density and composition: age-related and pathological changes in water and mineral content. J Bone J Surg. 1966:48:140-8. 
13. Laib A, Häuselmann HJ, Rüegsegger P. In vivo high resolution 3D-QCTof the human forearm. Technol Health Care Dec 1998;6:329-37.

14. Nishiyama KK, Macdonald HM, Buie HR, David A Hanley DA, Boyd SK. Postmenopausal women with osteopenia have higher cortical porosity and thinner cortices at the distal radius and tibia than women with normal aBMD: an in vivo HRpQCT study. J Bone Miner Res 2010:25: 882-90.

15. Burghardt AJ, Kazakia GJ, Ramachandran S, Link TM, Majumdar S.Age- and gender-related differences in the geometric properties and biomechanical significance of intracortical porosity in the distal radius and tibia $\mathrm{J}$ Bone Miner Res. 2010;25:983-93.

16. Burghardt AJ, Buie HR, Laib A, Majumdar S, Boyd SK. Reproducibility of direct quantitative measures of cortical bone microarchitecture of the distal radius and tibia by HR-pQCT Bone 2010;47:519-28.

17. Dice, LR. Measures of the amount of ecologic association between species. Ecology 1945; 26:297-302.

18. Cadet ER, Gafni RI, McCarthy EF, McCray DR, Bacher JD, Barnes KM, Baron J. Mechanisms responsible for longitudinal growth of the cortex: coalescence of trabecular bone into cortical bone. J Bone Joint Surg Am. 2003;85-A:1739-48.

19. Wang, Q, Wang, XF, Iuliano-Burns, S, Ghasem-Zadeh, A, Zebaze, R, and Seeman, E. Rapid growth produces transient cortical weakness: A risk factor for metaphyseal fractures during puberty. J Bone Miner Res 2010; 25:1521-6.

20. Dougherty G, Newman D. Measurement of thickness and density of thin structures by computed tomography. Med. Phys. 1999;26:1341-48

21. Prevrhal S, Engelke K, Kalander WA. Accuracy limits for the determination of cortical width and density: the influence of object size and CT imaging parameters. Phys. Med. Biol. 1999;44:751-64.

22. Yan Kang Y, Engelke K, Kalender WA. Interactive 3D editing tools for image segmentation. 2004; 8: 35-46

23. Treece GM, Gee AH, Mayhew PM, Poole KES. High resolution cortical bone thickness measurement from clinical CT data. Med Image Anal. 2010; 14: 276-90. 
24. Hangartner T.N. Thresholding technique for accurate analysis of density and geometry in QCT, PQCT and $\mu \mathrm{CT}$ images. J. Musculoskelet. Neuronal. Interact. 2007;7:9-16.

25. Mastmeyer A, Engelke K, Fuchs C, Kalender WA. A hierarchical 3D segmentation method and the definition of vertebral body coordinate systems for QCT of the lumbar spine. Med. Image Anal. 2006;10:560-77.

26. Buie HR, Campbell GM, Klinck RJ, MacNeil JA, Boyd SK. Automatic segmentation of cortical and trabecular compartments based on a dual threshold technique for in vivo micro-CT bone analysis. Bone 41; 2007: 505-15.

27. Goldenstein J, Kazakia GJ, Majumdar S. In vivo evaluation of the presence of bone marrow in cortical porosity in postmenopausal osteopenic women. Annals of Biomedical Engineering. 2010;38:235-46.

28. Melton LJ, Christen D, Riggs BL, Achenbach SJ, Müller R, van Lenthe GH, Amin S, Atkinson EJ, Khosla S . Assessing forearm fracture risk in postmenopausal women . Osteoporos Int 2010; 21:1161-9.

29. Tjong W, Kazakia GJ, Burghardt AJ, Majumdar S. The effect of voxel size on high-resolution peripheral computed tomography measurements of trabecular and cortical bone microstructure. Med. Phys. 2012;39:1892-1903.

30. Techawiboonwong A, Kwon Song HK, Leonard MB, Wehrli FW. Cortical bone water: In vivo quantification with ultrashort echo-time MR imaging. Radiology: 2008;248:824-33

31. Smith JW. Observations on the water content of bone. J Bone Joint Surg Am. 1964;46B:553-62.

32. Blitz RM, Pellegrino ED. The chemical anatomy of bone. J Bone Joint Surg Am. 1969;51: 456-66

33. Engelke K, Stampa B, Timm W, Dardzinski B, de Papp A, Genant HK, T. Fuerst T, (2011). Short-term in vivo precision of BMD and parameters of trabecular architecture at the distal Forearm and tibia. Osteoporos Int. 2011;23: 2151-2158.

34. Ruffoni D, Fratzl P, Roschger P, Klaushofer K, Weinhmer R. The bone mineralization density distribution as a fingerprint of the mineralization process. Bone 2007; 40:1308-1940.

35. Parfitt AM, Rao DS, Stanciu J, Villanueva AR, Kleerekoper M, and B. Frame B Irreversible Bone Loss in Osteomalacia Comparison of Radial Photon Absorptiometry with Iliac Bone Histomorphometry during Treatment J. Clin. Invest. 1985;76: 24032412 
36. Boivin GY, Chavassieux PM, Santora AC, Yates JY, Meunier PJ. Alendronate increases Bone strength by increasing the mean degree of mineralization of bone Tissue in osteoporotic women. Bone 2000; 27:687-694

37. Akkus O, Polyakova-Akkus A, Adar F, Schaffler M. Aging of microstructural compartments in human compact bone. J Bone Miner Res 2003;18:1012-1019.

38. Zebaze RM, Jones AC, Pandy MG, Knackstedt MA, Seeman A. Differences in the degree of bone tissue mineralization account for little of the differences in tissue elastic properties. Bone 2011; 48:1246-51.

39. Fajardo RJ, Cory E, Patel ND, Nazarian A, Laib A, Manoharan RK, Schmitz JE, DeSilva JM, MacLatchy LM, Snyder BD, Bouxsein ML. Specimen size and porosity can introduce error into microCT-based tissue mineral density measurements. Bone $2009 ; 44: 176-84$. 


\section{Figures legends}

Figure 1 (A) A grey scale image of a distal radius cross-section acquired using high resolution peripheral quantitative computed tomography. The Arm (white rectangle) originates at ' $\mathrm{C}$ ' and extends to $\mathrm{P}_{0}$. (B) The magnified Arm shows its contents of background, bone edges and compartments. (C) The attenuation profile. The coordinates define the bone edges and so the compartments. These coordinates are identified from moving referents. The first, $\mathrm{P}_{1}$, has a y value equal to the background attenuation and an $\mathrm{x}$ value corresponding to the maximum $\mathrm{y}$ attenuation. From $\mathrm{P}_{1}$, the smallest circle intersecting the attenuation profile curve is ' $\mathrm{K}$ ', the beginning of the background/bone interface. The distance between ' $\mathrm{C}$ ' and ' $\mathrm{K}$ ' is the radius of the bone cross section at that angle. Radii at $12 \mathrm{pm}, 3 \mathrm{pm}, 6 \mathrm{am}$, and 9 am are identified by rotating 90 degrees and applying the same algorithm. The smallest of the radii $\left(\mathrm{R}_{\mathrm{i}}\right)$ is used to automatically quantify the width of Arm that will be used for segmentation. After identification of ' $\mathrm{K}$ ', the moving referents $\mathrm{P}_{1}, \mathrm{P}_{2}, \mathrm{P}_{3}$ identify coordinates that define the beginning ' $\mathrm{M}$ ' and end ' $\mathrm{N}$ ' of compact-appearing cortex (inset). Between ' $\mathrm{N}$ ' and ' $\mathrm{O}$ ', the beginning of the trabecular compartment, is the transitional zone. The zone ' $\mathrm{K}$ ' to ' $\mathrm{M}$ ', at the background/bone interface is also a transitional zone which is excluded during segmentation to quantify porosity (see text and figure in Appendix).

Figure 2. A scanning electron microscopic image of the subtrochanteric showing the compact-appearing cortex, transitional zone and trabecular compartment. The density profile curve produced has two plateaus; one corresponding to the compact-appearing cortex and one corresponding to the trabecular compartment. Between these plateaus is a descending $\mathrm{S}$ shaped curve or transition between the two plateaus. This is the transitional zone. The y-axis is the density profile curve expressing the mineralized bone area as the percentage of total area within each column (black dotted rectangles).

Figure 3. Left panels: Correlations between HRpQCT and the gold standard (micro-CT) for total cross sectional area (T-CSA), compact-appearing cortex, transitional zone, and medullary areas. Right panels: Corresponding Bland and Altman plots at the distal radius.

Figure 4. Left panels: Correlations between HRpQCT and gold standard (microCT) and for porosity of the compact-appearing cortex and transitional zone. Right panels: Corresponding Bland and Altman plots at the distal radius.

Figure 5. Left panels: Correlations between HRpQCT and the gold standard (microCT) for total cross sectional area (T-CSA), compact-appearing cortex, transitional zone, and medullary areas. RMSE were $11.49 \mathrm{~mm}^{2}, 1.64 \mathrm{~mm}^{2}, 2.27 \mathrm{~mm}^{2}, 14.79 \mathrm{~mm}^{2}$ at the distal tibia Right panels: Corresponding Bland and Altman plots at the distal 
tibia. The area of the compact cortex was underestimated by $12.09 \mathrm{~mm}^{2}(13.4 \%)$ whereas the area of the transitional zone was overestimated by $3.17 \mathrm{~mm}^{2}$

Figure 6. Left panels: Correlations between HRpQCT and gold standard (micro-CT) and for porosity of the compact-appearing cortex and transitional zone (All $\mathrm{P}<0.05$ ) . RMSE were 2.41 and $1.39 \%$ respectively at the distal tibia. Right panels: Corresponding Bland and Altman plots at the distal tibia. There was no over or underestimation of the porosity of the compact-cortex. Porosity of the transitional zone was underestimated by $2.38 \%$

Figure 7. Upper row: The correlation between total cross sectional area (T-CSA) measured manually (i.e., directly) and by StrAx1.0 $\left(\mathrm{r}^{2}=0.99\right)$ and the Bland-Altman plots for the distal tibia and distal radius. Lower row: The correlation between compact-appearing cortical area measured manually and by StrAx $1.0\left(r^{2}=0.94\right.$ to 0.97 ) and the Bland-Altman plots for the distal tibia and distal radius.

Figure 8. In vivo comparison of $\operatorname{StrAx} 1.0$ and Scanco method. Distal radius- Left panels: A, B, C show correlations between StrAx1.0 and Scanco methods for total cross sectional area (T-CSA), compact-appearing cortex, transitional zone, medullary areas, and porosity of the compact cortex. Right panels: Bland and Altman plots show that T-CSA measured by Scanco was smaller than measured by StrAx1.0 and this difference was independent of bone size. Compact-cortex and transitional zone areas by Scanco were also smaller than measured by StrAx1.0 and the greater the area of compact-cortex or transitional zone, the larger the compartment size measured by Scanco compared to StrAx1.0 (see text). In D, the left panel shows that porosity measured by Scanco and StrAx1.0 in the compact-appearing cortex correlated modestly. Bland and Altman (right panel) show that porosity measured by Scanco was $16.8 \%$ lower than measured by StrAx 1.0 and the higher the 'true' porosity (average of both values), the greater the underestimate.

Figure 9. The upper middle panel (B) shows an original total HRpQCT image of the distal radius and the external and internal contours as delineated by the standard manufacturer software (Scanco). The lower middle panel shows the same total image with the background color-coded blue. The compact-cortex, transitional zone, and trabecular compartments are color-coded green, red and yellow, respectively. Left (A) and right (B) panels are magnified regions showing differences in segmentation between StrAx1.0 and Scanco. Segmenting by the Scanco algorithm in this case allocates cortical porosity and cortical fragments to the medullary (trabecular) compartment. While manual correction is recommended by the manufacturer, it is impractical and difficult to perform. The segmentation error was avoided by StrAx1.0. 


\section{Appendices}

I Automatic calculation of the attenuation produced by background (P) and fully mineralized bone (B) for quantifying porosity in in vitro images

Automated retrieval of values is done for high resolution images such as scanning electron microscopic (SEM) images when the attenuation values corresponding to background $(\mathrm{P})$ and fully mineralized bone matrix $(\mathrm{B})$ are unknown. This retrieval is not performed for HRpQCT images as these values are computed from phantom calibration.

A pixel (or voxel) within the subperiosteal envelope that contains only void has the same attenuation as background (P). Background can be air in in vitro $\mathrm{CT}$ images of bone, or polymethyl methacrylate (PMMA) for SEM images. Pixels in the background are heterogeneous and may contain a variable proportion of fat, water, air, and muscle. Thus, not all voxels can be regarded as being representative of the background (i.e., the attenuation of the voxel containing only void). To retrieve the value that corresponds to background, during segmentation all pixels in the Arm located before ' $\mathrm{K}$ ' are retrieved and considered as background voxels (see figure 1 main text). Once all pixels with their attenuation (or intensity) values have been retrieved, a frequency distribution curve of pixels is computed. The attenuation (or intensity) of the pixels at the $90^{\text {th }}$ percentile is assumed to be high enough to capture the background value, yet not so high as to reflect noise.

Similarly, finding a pixel (or voxel) occupied by mineralized bone matrix in in vitro specimens is achievable automatically provided that the image is of high resolution such as an SEM image. The attenuation produced by pixels between Haversian canals is assumed to reflect only mineralized bone matrix free of void. As the entire bone image is reconstructed using several thousands of pixels, it is assumed that some pixels are located within the inter-Haversian region. The distance between two contiguous Haversian canals is about $200 \mu \mathrm{m}$ [ $1 \& 9$, main text]. These voxels with the highest attenuation form the upper tail of the frequency distribution curve. To identify them, all pixels within the periosteal envelope are retrieved with their attenuation values and the value corresponding to the $90^{\text {th }}$ percentile is computed and assumed to be high enough to capture fully mineralized bone matrix, yet not so high as to reflect noise.

\section{II- Potential errors in quantification of porosity introduced by heterogeneity in bone matrix mineralization}


Heterogeneity in the completeness of secondary mineralization can influence the accuracy of measurement of porosity in an individual. However, regardless of the heterogeneity of mineralization within an individual, there are small, if any, differences in the mean degree of mineralization between healthy individuals of varying sexes, races, and ages after completion of longitudinal growth [Appendix ref 1,2, 38 main text]. Hence, in these circumstances, there is little, if any, influence of heterogeneity upon differences in porosity calculated by StrAx1.0 between individuals.

To obtain an accurate measure of porosity in an individual, the algorithm assumes that the degree of matrix mineralization is constant and about maximal in all pixels (or voxels). However, the degree of completeness of secondary mineralization varies point by point within the bone. We examined the magnitude of the heterogeneity in mineralization using SEM images at $5 \mu \mathrm{m}$ resolution. At this level of resolution pixels overlying mineralized bone matrix with different grey levels are clearly distinguishable from voids. From the quantified level of heterogeneity, we estimated the size of a potential error that mineralization heterogeneity may introduce in the quantification of porosity.

Mineralization was measured and its heterogeneity was assessed in 520 consecutive random points from SEM images of cortical samples from trochanters obtained at post-mortem from 12 Caucasian females (mean age: 69 years; range: 29 to 85) [31 main text]. We then simulated a resolution of $42 \mu \mathrm{m}$ by manually randomly selecting $42 \mu \mathrm{m}$ square regions in SEM images in 4 post-mortem samples and measuring their average grey level values. A total of 106 regions were randomly selected. Resolutions from 5 to $42 \mu \mathrm{m}$ are those used when an entire cross-section of bone is reconstructed from SEM images to quantify porosity in vitro. Finally, we simulated a resolution of $82 \mu \mathrm{m}$ to examine the heterogeneity in mineralization at a resolution used in HR-pQCT imaging. This heterogeneity was calculated and used to estimate the error that may arise in the quantification of porosity.

As shown in Appendix figure 3 and the legend, across resolutions, the mean mineralization density or grey level value was, on average, $\sim 10 \%$ below the maximum, with the greatest deviation rarely more than $20 \%$ below the maximum. Thus, to ensure that pixels (or voxels) containing only mineralized bone matrix at different degrees of completeness of mineralization are not erroneously said to contain pores, all voxels within $20 \%$ of the maximum mineralization value (i.e., within $20 \%$ of the attenuation corresponding to B) are excluded. Porosity is then calculated, but only in voxels that have an attenuation between 0 and $80 \%$ of the maximum value. Under these circumstances, there is a $<5 \%$ likelihood that pixels containing only submaximally mineralized bone tissue would be erroneously deemed to contain pores. In the remaining voxels, the magnitude of the accuracy error due to bone matrix mineralization heterogeneity is $\sim 10 \%$, that is, the porosity of a voxel can be estimated with on average $\sim 90 \%$ accuracy.

In SEM images, about $50 \%$ of the cortex is made of pixels containing mineralized bone matrix at various stage of mineralization. Different degrees of secondary mineralization are visible as different degrees of whiteness and greyness. These are excluded from measuring porosity by StrAx1.0. Measurement of BMD or BMC does 
not exclude these areas. The remaining $50 \%$ of pixels are composites (i.e., partly void, partly bone matrix) and these are comprised of $\sim 50 \%$ mineralized bone matrix and $\sim 50 \%$ void. Assuming that the $50 \%$ mineralized bone matrix within a pixel is on average $10 \%$ submaximally mineralized, the error in estimating the void area in that pixel is $5 \%$ (i.e., $10 \%$ of $50 \%$ ); i.e., the true porosity is $45 \%$ not $50 \%$. To calculate the error in the quantification of the total porosity in the total cortex, not all pixels are subject to that error, e.g., empty pixels and pixels above $80 \%$ of the maximum mineralization are not subject to this error. In advanced age, when porosity is high, composite pixels not subject to the error account for about $60 \%$ of all pixels within the cortex. Thus, this $5 \%$ error occurs in only $60 \%$ of the cortical area overestimating porosity by $3 \%$ (i.e. $5 \%$ of, $60 \%$ ).

In HRpQCT images in vivo, about $50 \%$ of the cortex is made of voxels containing mineralized bone matrix at various stage of mineralization. These voxels are excluded from the calculation of porosity. The difference between cortical BMC or $\mathrm{BMD}$, and porosity quantified by StrAx1.0 is largely due to the exclusion of these voxels which are about $50 \%$ of all cortical voxels. The remaining $50 \%$ of voxels which are composite voxels and thus used in the quantification of porosity, are comprised on average of $50 \%$ mineralized matrix volume and $50 \%$ void space. Assuming that the $50 \%$ mineralized bone matrix volume within the voxel is on $10 \%$ submaximally mineralized, then the error in estimating the void fraction of the voxel is $5 \%$ (i.e., $5 \%$ of $50 \%$ ). In advanced age, when porosity is high, composite voxels whhch are subject to the potential error account for $50 \%$ of all voxels within the cortex. Thus, this $5 \%$ error occurs in only $\sim 50 \%$ of all cortical voxels resulting in an overestimation of porosity of $\sim 2.5 \%$ (i.e., $5 \%$ of the $50 \%$ o).

Porosity may also be underestimated. This may occur when voxels with attenuation value within $20 \%$ of the maximum contain a pore and are disregarded by StrAx 1.0 assuming they only contain submaximally bone matrix. The size of this error depends on the number of voxels which can contain a pore so small that it decreases the maximum attenuation to a value less than $20 \%$. This error does not occur in SEM image as the resolution is high enough to clear discern pores. The smallest Haversian canal is $\sim 15$ to $20 \mu \mathrm{m}$ in size. In HRpQCT images, it is possible that a voxel may contain maximally mineralized bone matrix and a small pore. However, for this to occur, the pore should be $\sim 24 \mu \mathrm{m}$ or less in size. Based on direct quantification of porosity in 2117 pores across 24 samples, pores of this size represented $\sim 11 \%$ of all pores and accounted for $\sim 3 \%$ of total porosity. If these pores are ignored porosity will be underestimated by $\sim 3 \%$.

Thus, porosity may be overestimated by $\sim 2.5 \%$ due to submaximally mineralized voxels being considered as maximally mineralized in voxels composite voxels included in the calculation of porosity. At the same time porosity may also be underestimation by $\sim 3 \%$ due to the exclusion of pores of $24 \mu \mathrm{m}$ or less,. This simultaneous over- and under estimation would result in an overall accurate quantification of porosity. 


\section{Appendix references}

1. Roschger P, Gupta HS, Berzlanovich A, Ittner G, Dempster DW, Fratzl P, Cosman F, Parisien M, Lindsay R, Nieves JW, Klaushofer K. Constant mineralization density distribution in cancellous human bone. Bone. $2000 ; 32(3): 316-23$.

2. Tissani S, Öhman C, Baruffaldi F, Baleani M, Viceconti M. Volume to density relation in adult human bone tissue. J Biomech 2011;44:103-108. 


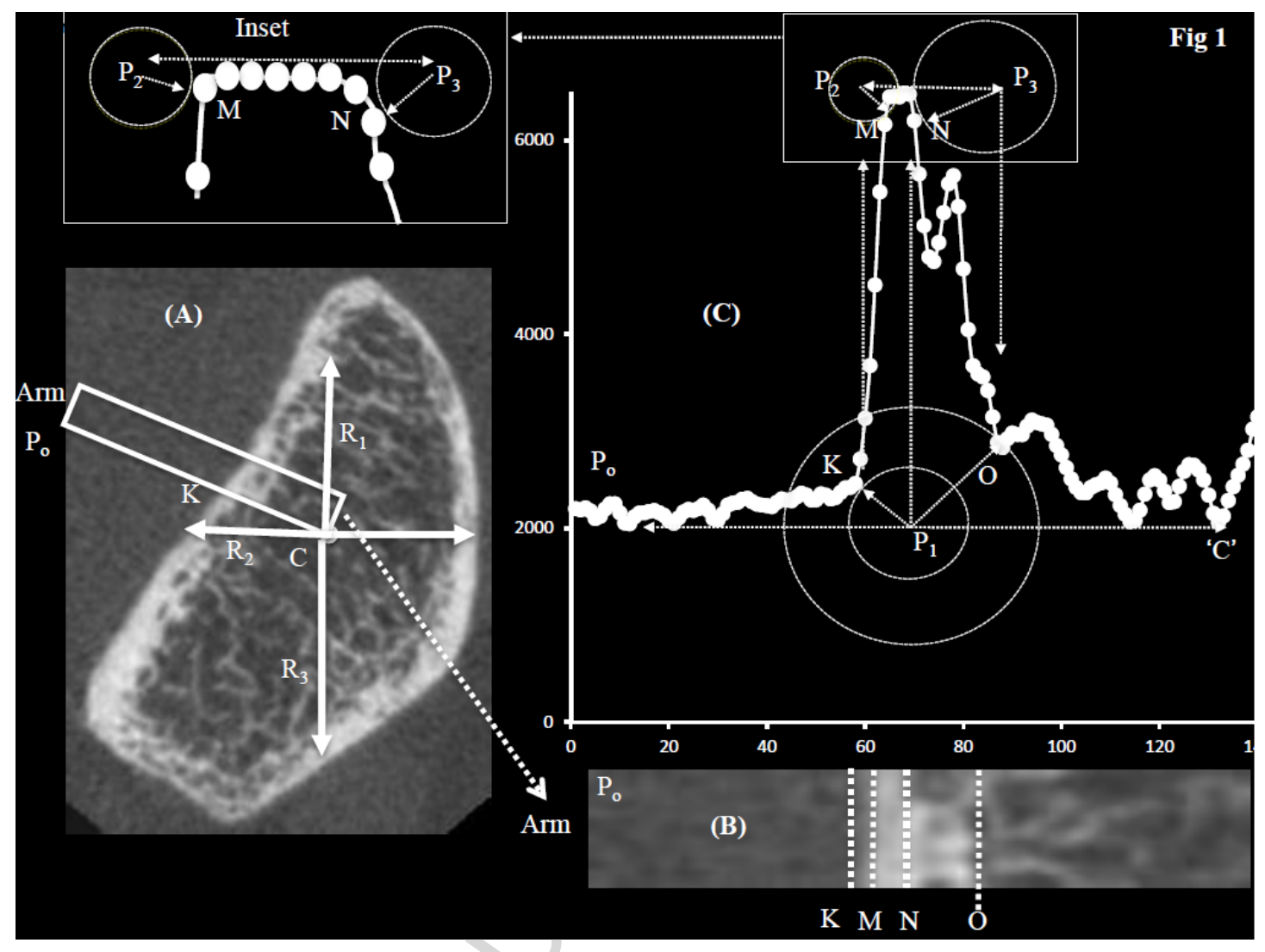




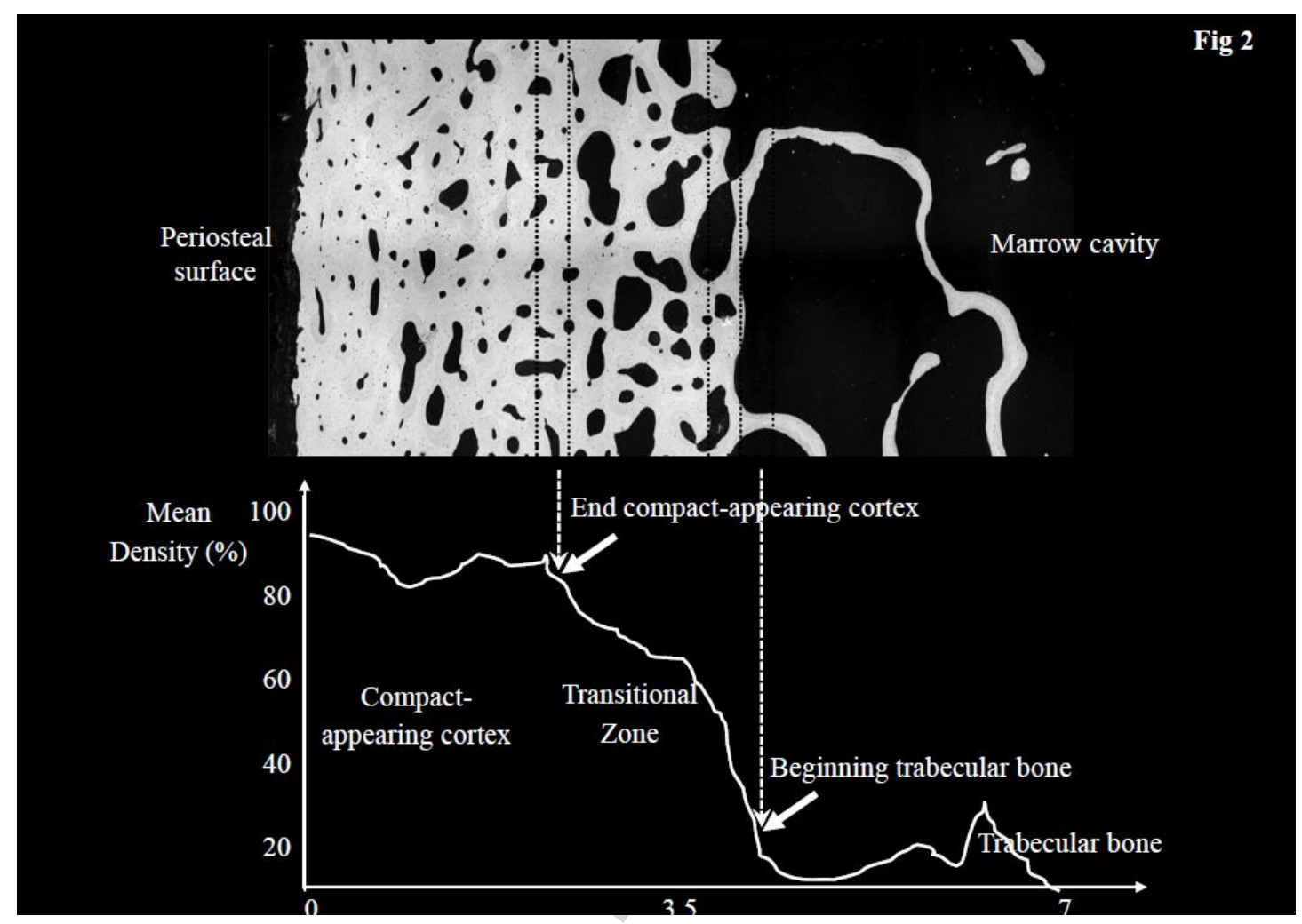


Distal Radius

Fig 3

Total cross sectional area
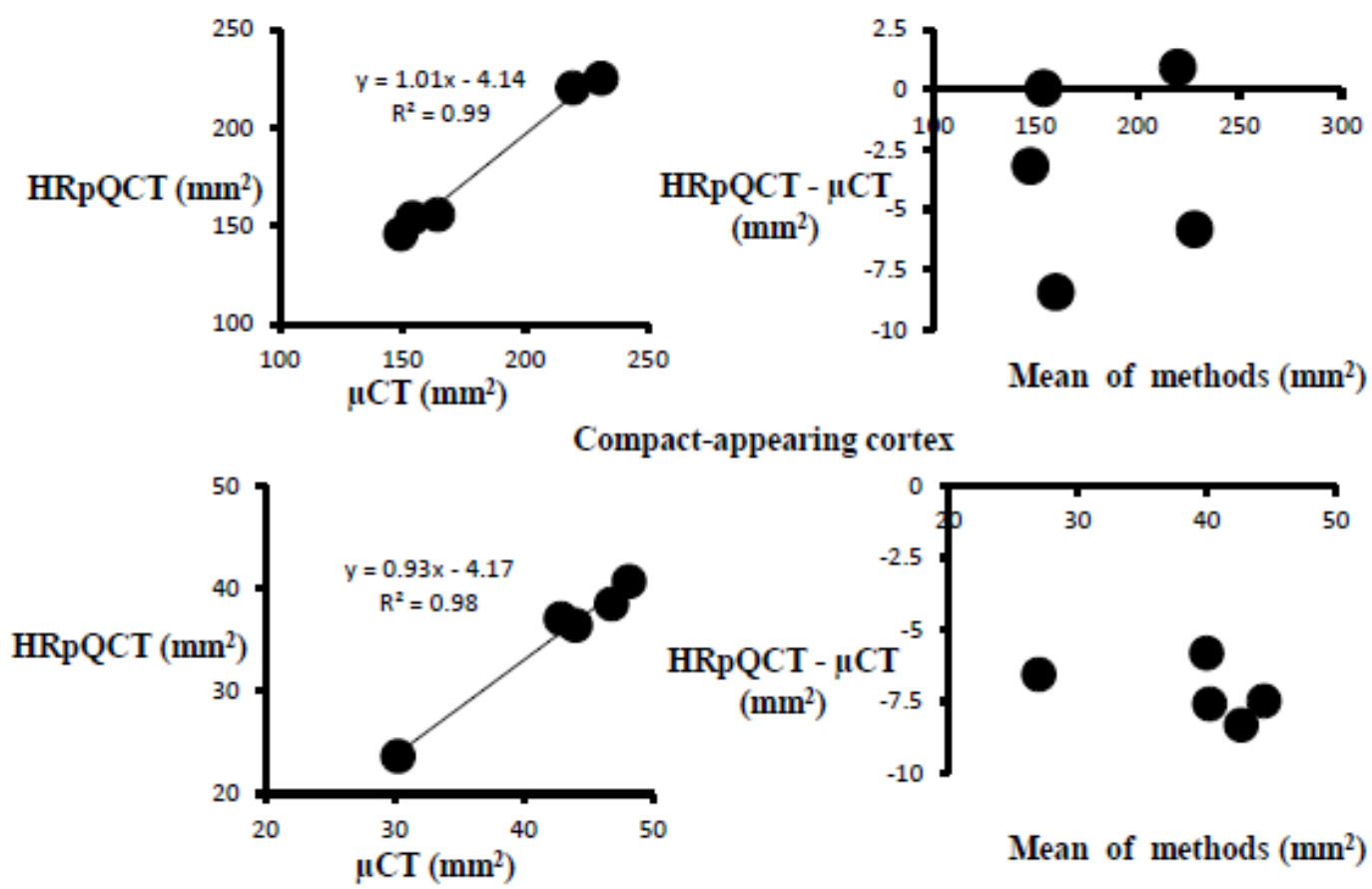

Mean of methods $\left(\mathrm{mm}^{2}\right)$

Transitional zone
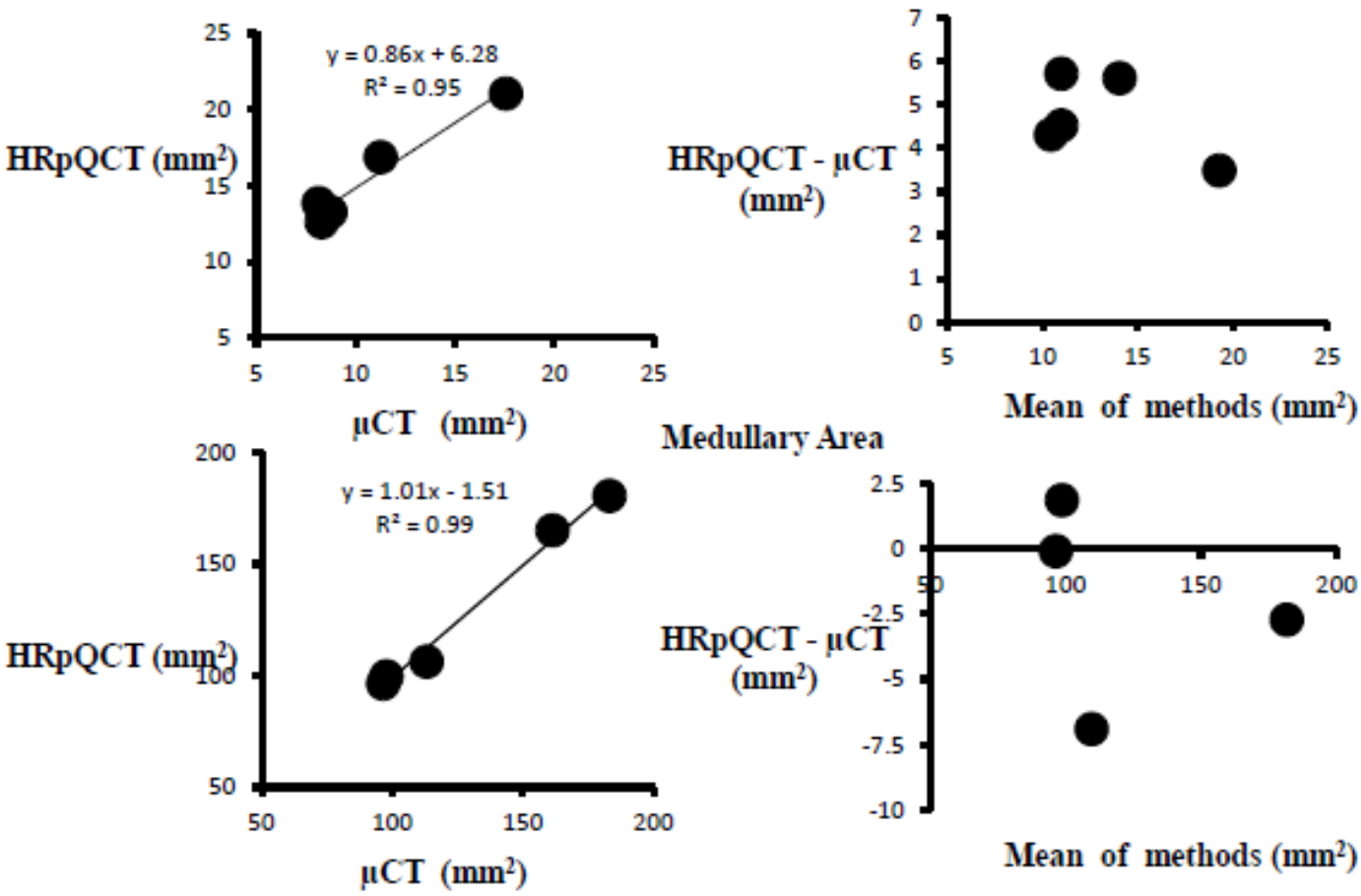

Mean of methods $\left(\mathrm{mm}^{2}\right)$ 
Distal Radius
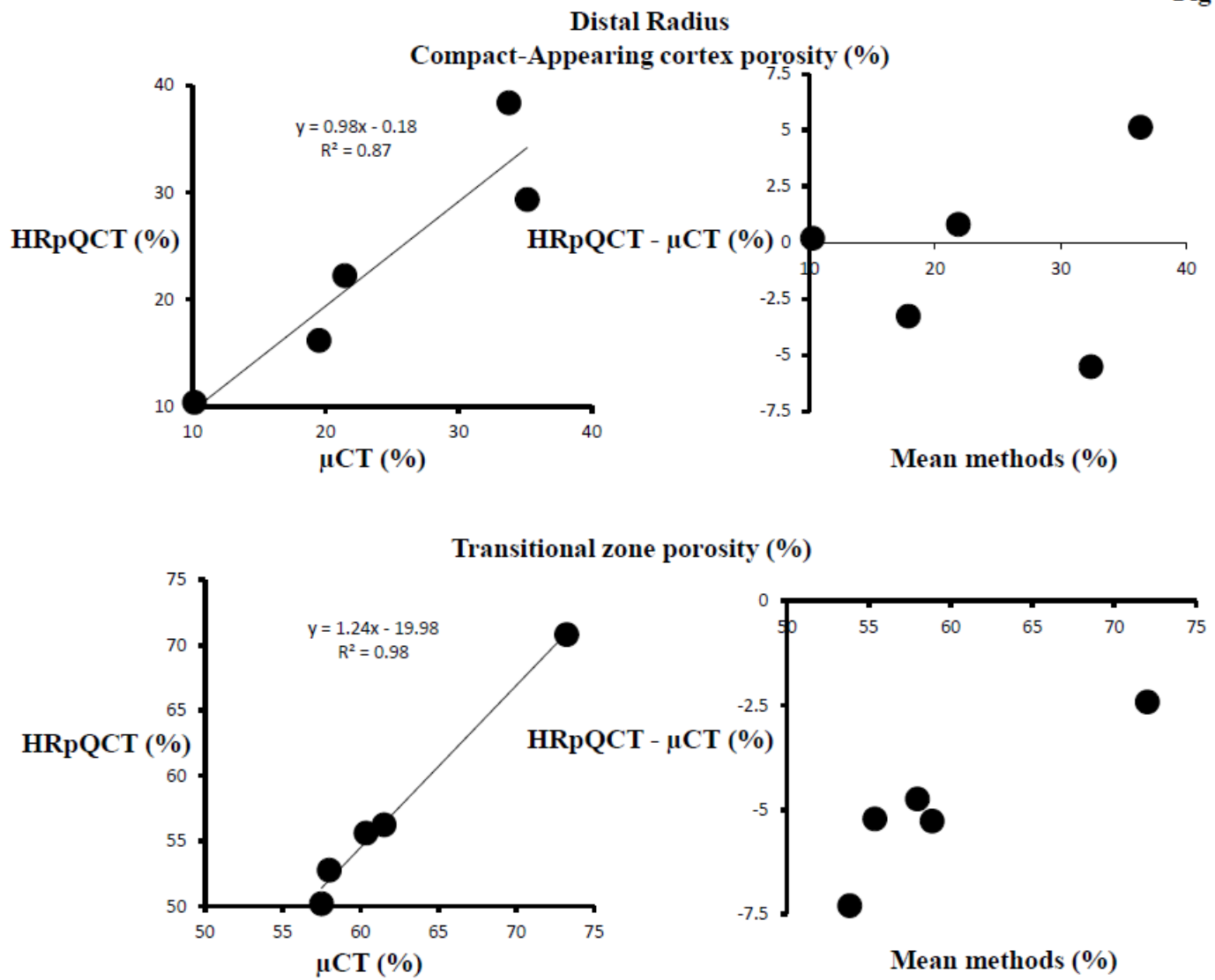
Distal Tibia

Fig 5

Total cross sectional area
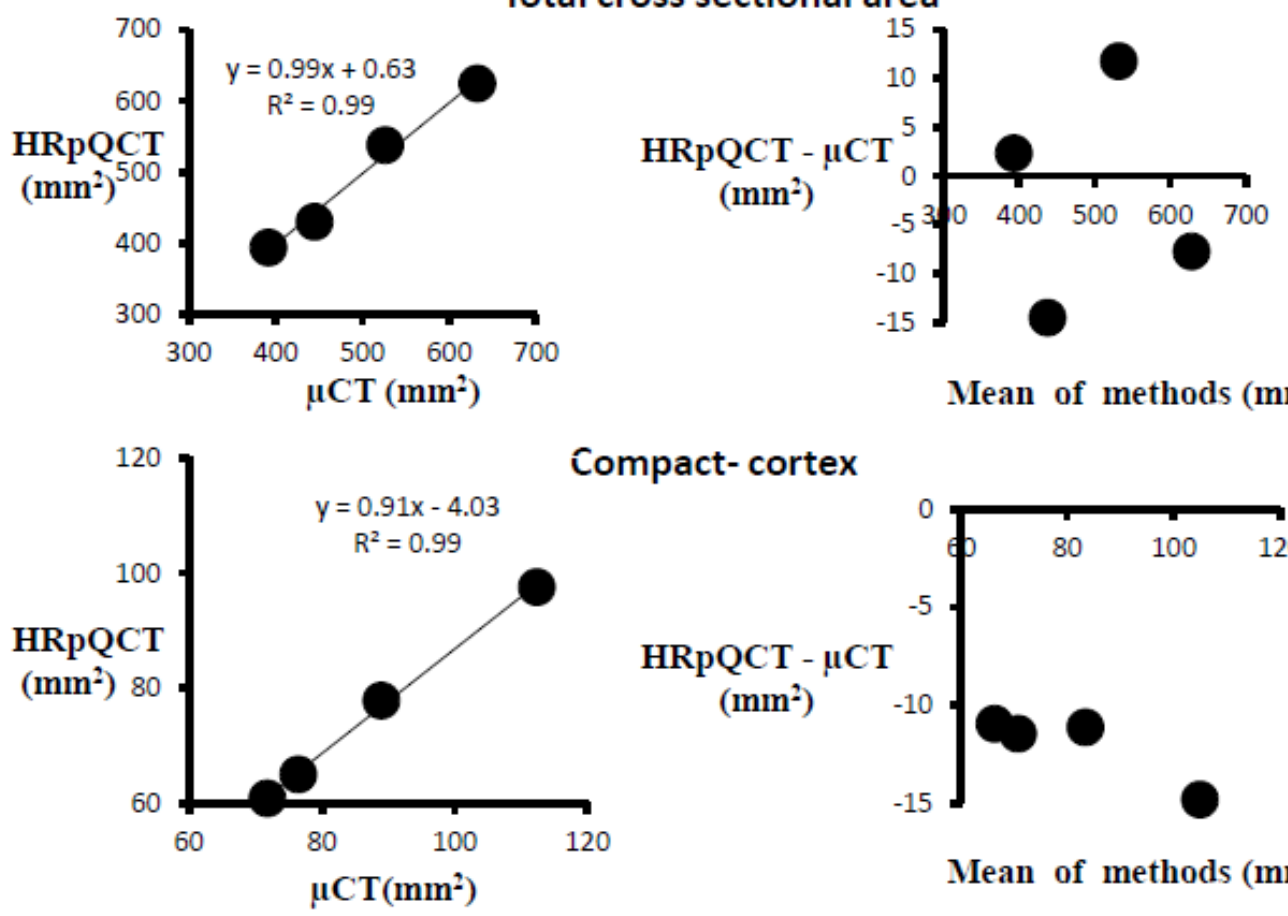

Mean of methods $\left(\mathrm{mm}^{2}\right)$

Compact- cortex
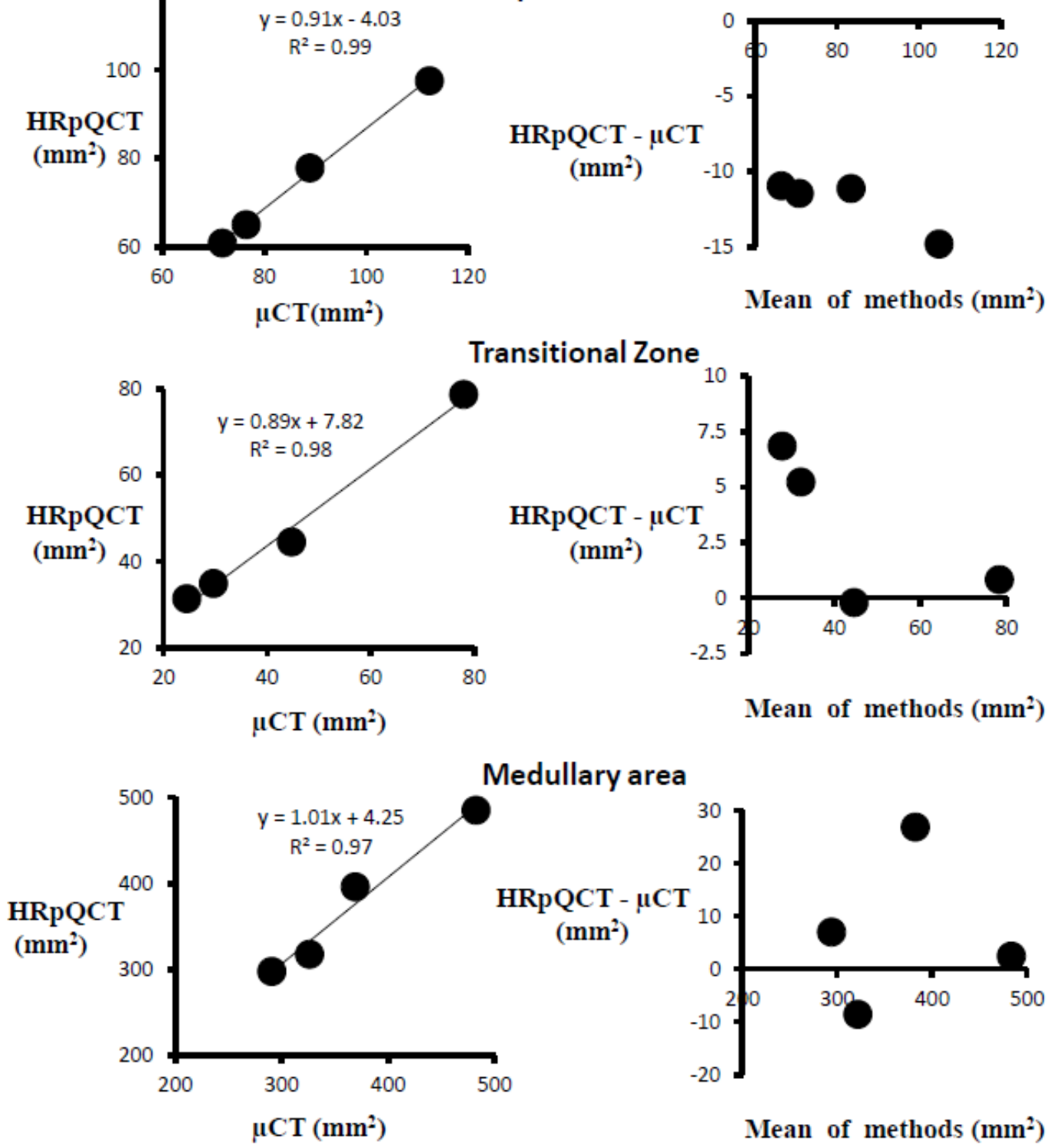
Distal Tibia

Fig 6

Compact-Appearing cortex porosity (\%)
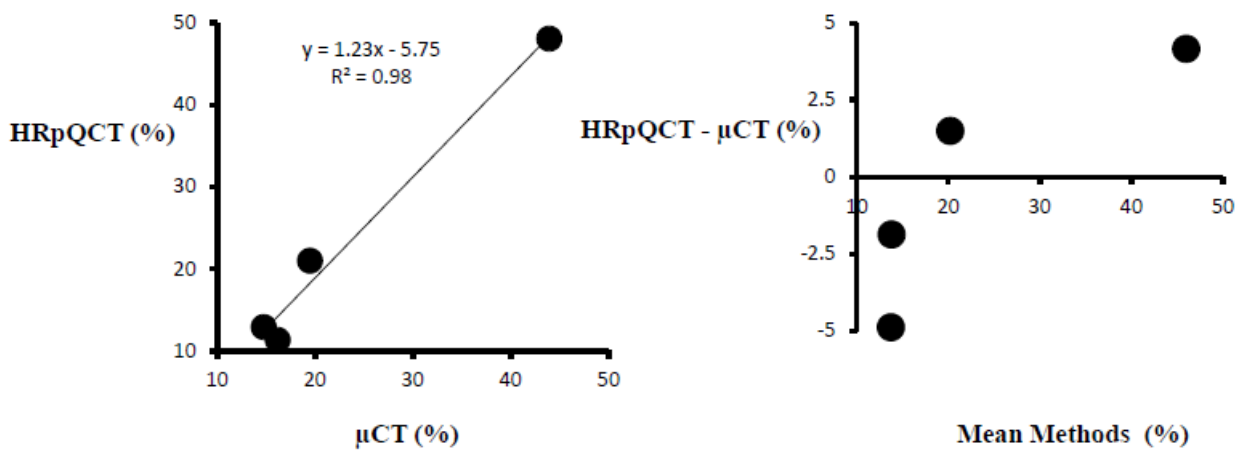

Mean Methods (\%)

Transitional zone porosity (\%)

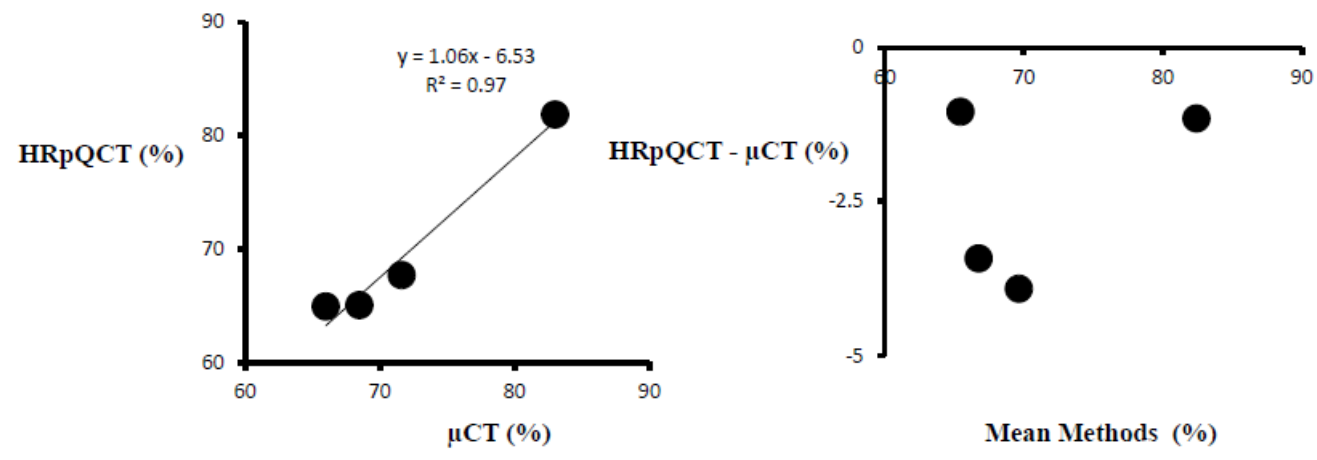



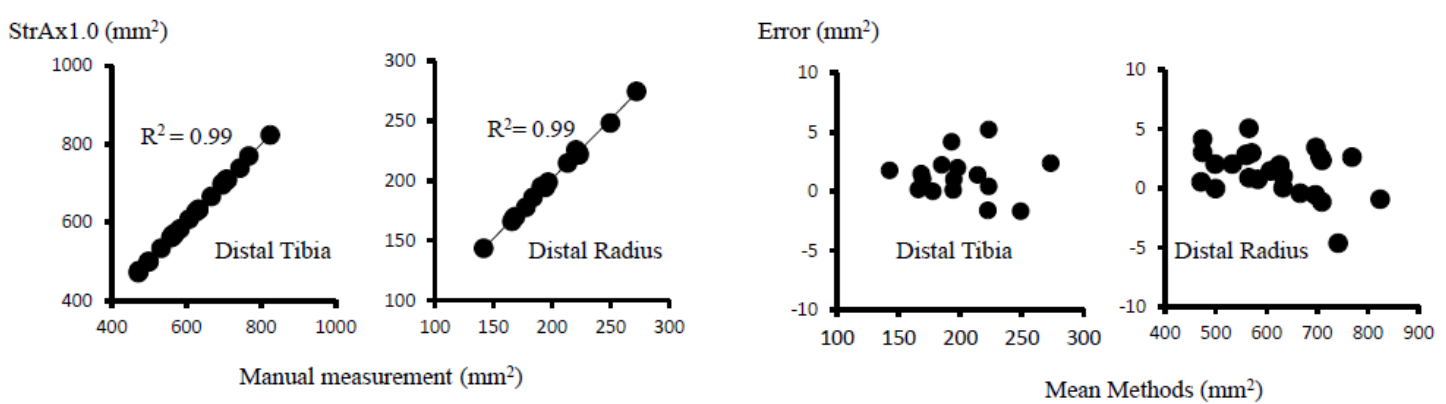

Compact-appearing cortex (mm²)
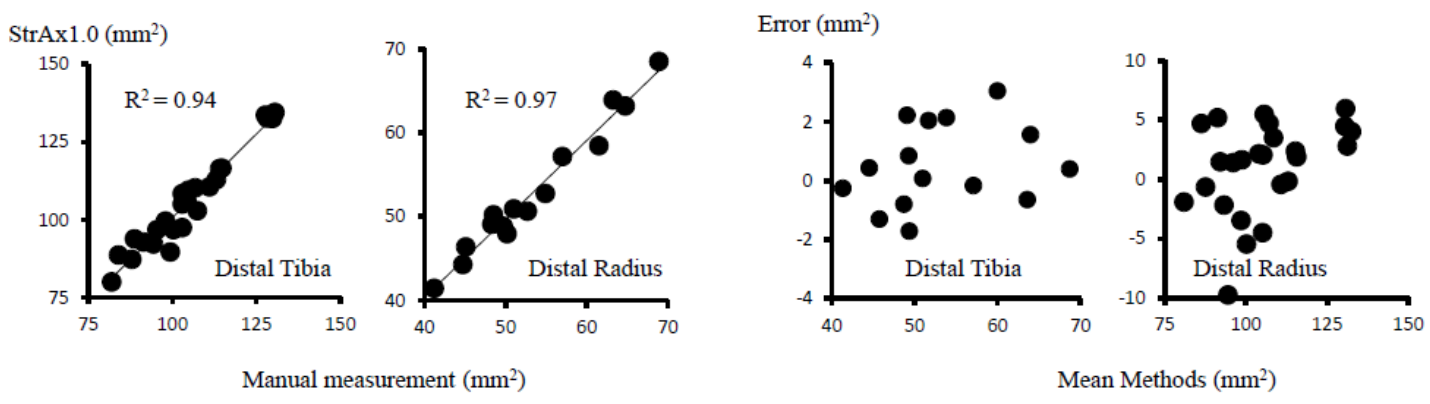
Fig 8

Difference

Scanco

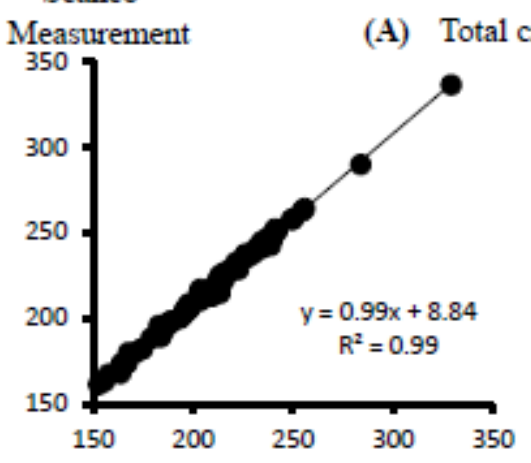

(B) Compact-appearing cortex $\left(\mathrm{mm}^{2}\right)$
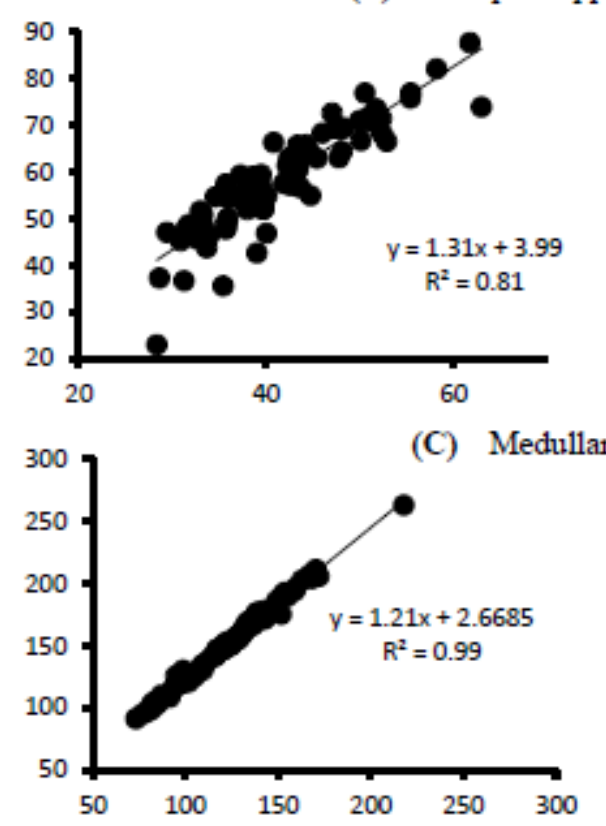

(D) Porosity (\%)

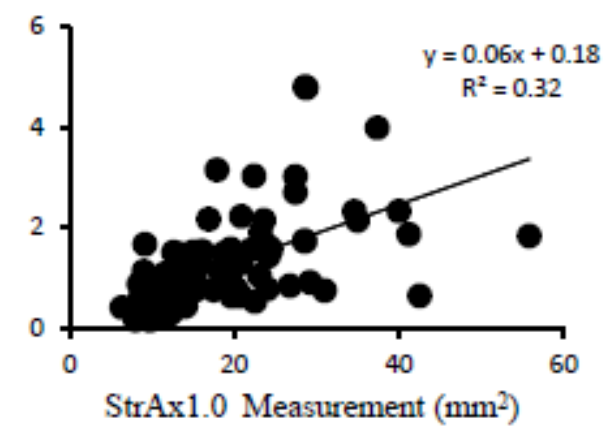

(Scanco - StrAx1.0)

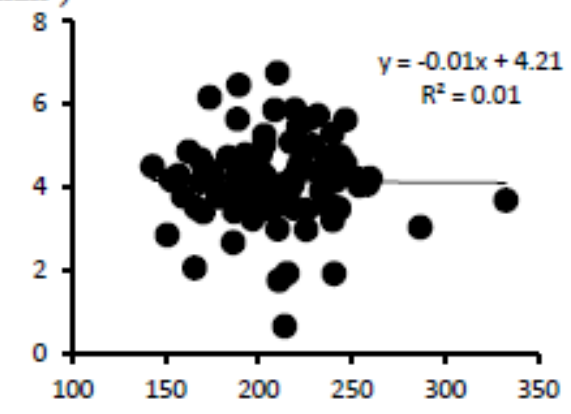

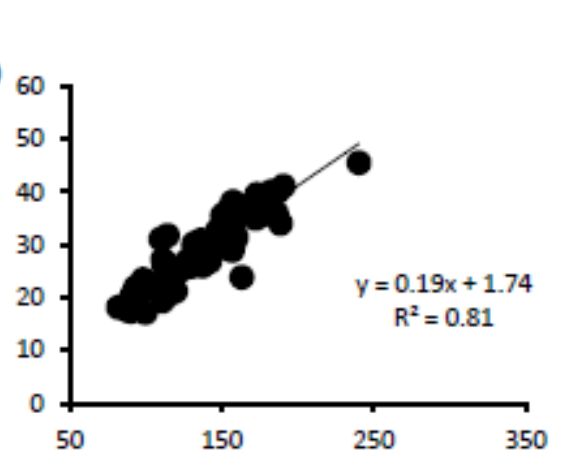

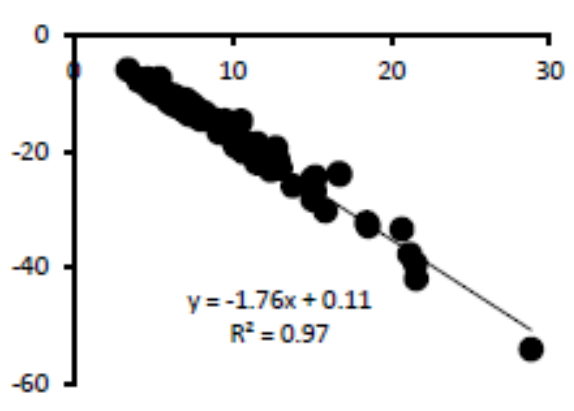

Mean of Methods $\left(\mathrm{mm}^{2}\right)$ 


\section{ACCEPTED MANUSCRIPT}
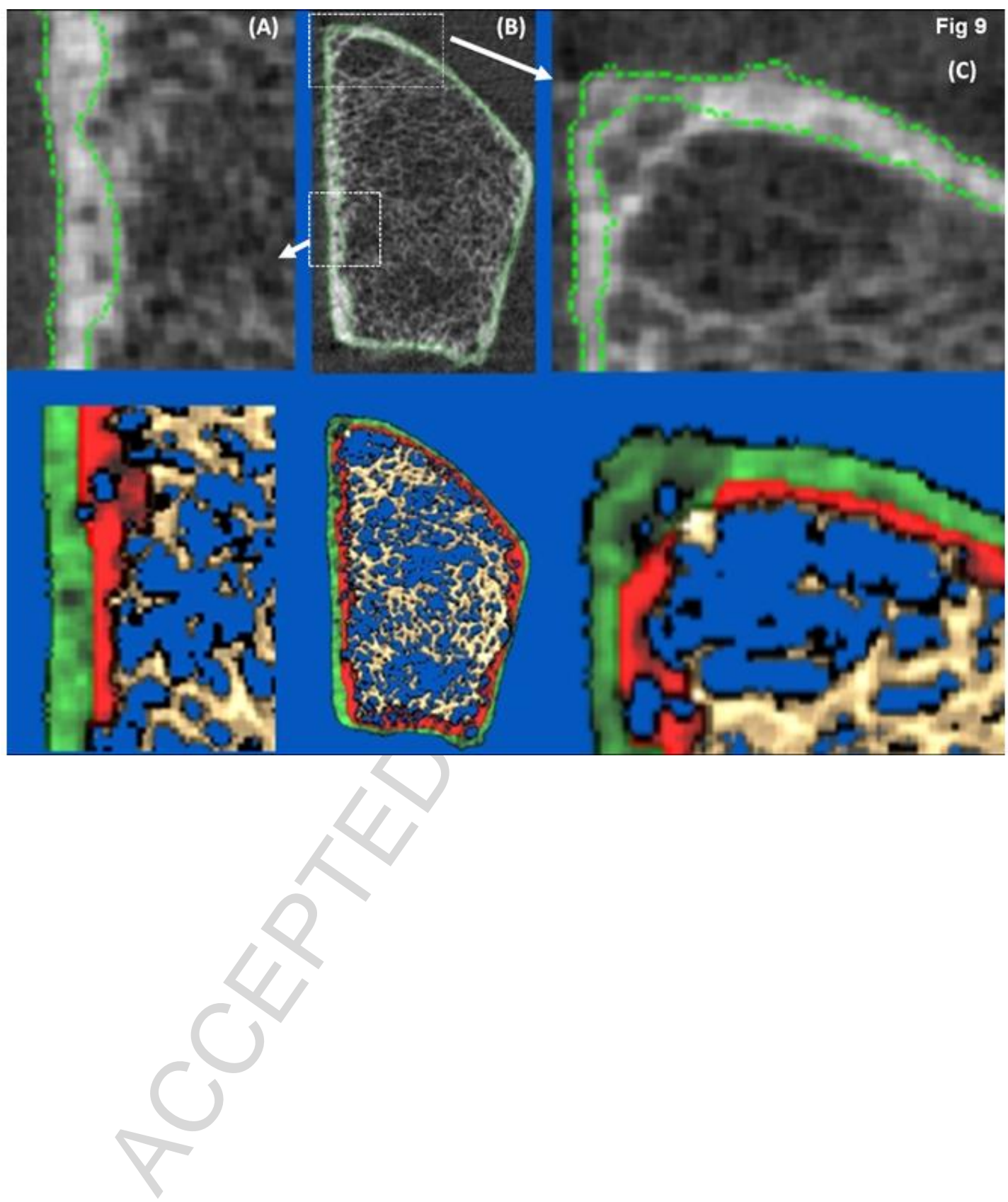
Table 1. Regression analyses at the proximal femur (subtrochanteric region) between gold standard scanning electron microscopy (SEM) analysed manually and StrAx1.0. Measurements include total cross sectional area (T-CSA), compactappearing cortex, transitional zone, trabecular compartment, and porosity in that SEM images, and in an HRpQCT images ex vivo of a region similar to that of SEM image.

\begin{tabular}{|c|c|c|c|c|c|}
\hline & & $\mathbf{R}^{2}$ & Slope & intercept & RMSE \\
\hline \multirow[t]{2}{*}{ T-CSA $\left(\mathrm{mm}^{2}\right)$} & StrAx1.0-SEM & 0.99* & 1.04 & -32.00 & 6.07 \\
\hline & StrAx1.0 HRpQCT & $0.93 *$ & 1.66 & -442.4 & 25.96 \\
\hline \multirow{2}{*}{$\begin{array}{l}\text { Compact-appearing } \\
\text { Cortex }\left(\mathrm{mm}^{2}\right)\end{array}$} & StrAx1.0-SEM & $0.98 *$ & 1.08 & -33.74 & 12.00 \\
\hline & StrAx1.0 HRpQCT & $0.91 *$ & 0.99 & 9.17 & 27.63 \\
\hline \multirow{2}{*}{$\begin{array}{c}\text { Transitional Zone } \\
\left(\mathbf{m m}^{2}\right)\end{array}$} & StrAx1.0-SEM & $0.98 *$ & 0.69 & 15.69 & 3.37 \\
\hline & StrAx1.0 HRpQCT & $0.92 *$ & 0.88 & 9.86 & 10.3 \\
\hline \multirow{2}{*}{$\begin{array}{l}\text { Medullary Area } \\
\qquad\left(\mathrm{mm}^{2}\right)\end{array}$} & StrAx1.0-SEM & $0.97^{*}$ & 1.11 & -22.74 & 10.84 \\
\hline & StrAx1.0 HRpQCT & $0.96 *$ & 1.35 & -112.9 & 13.22 \\
\hline \multirow{2}{*}{$\begin{array}{l}\text { Compact-appearing } \\
\text { cortex porosity }(\%)\end{array}$} & Ax1.0 -SEM & $0.97 *$ & 1.01 & -0.32 & 1.85 \\
\hline & 1.0-HRpQCT & $0.97 *$ & 1.24 & -1.12 & 2.30 \\
\hline
\end{tabular}

$*$ denotes $\mathrm{p}<0.05$. RMSE denotes root mean square error 
Table 2 In vivo reproducibility error expressed as RMSCV(\%) of StrAx1.0 measurement of compact-cortex, transitional zone, and medullary areas; and quantification of porosity at the distal radius.

\begin{tabular}{ccc}
\hline & Segmentation & Porosity \\
\hline T-CSA & 1.08 & \\
Compact-Cortex & 3.98 & 2.92 \\
Transitional Zone & 1.88 & 0.54 \\
Medullary Area & 2.96 & \\
\hline
\end{tabular}




\section{Research Highlights}

1. StrAx1.0 accurately and reproducibly segments and quantifies cortical and trabecular compartments, and the transitional zone or cortico-trabecular junction, a region of intense remodeling from high resolution images using curve profile analysis.

2. Segmentation of the transitional zone minimizes erroneous apportioning of cortical fragments and porosity to the trabecular compartment or thick densely connected trabeculae to the cortical compartment.

3. Porosity is accurately quantified as the averaged void volume fraction in all voxels within each compartment rather than just counting void voxels.

4. StrAx1.0 accurately and reproducibly quantifies porosity below, as well as above, the voxel size of 82 microns, from HRpQCT images. 


\section{University Library}

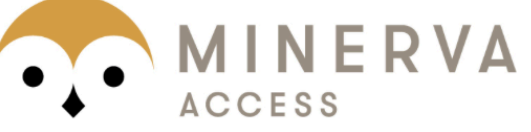

A gateway to Melbourne's research publications

Minerva Access is the Institutional Repository of The University of Melbourne

Author/s:

Zebaze, R;Ghasem-Zadeh, A;Mbala, A;Seeman, E

Title:

A new method of segmentation of compact-appearing, transitional and trabecular compartments and quantification of cortical porosity from high resolution peripheral quantitative computed tomographic images

Date:

2013-05-01

Citation:

Zebaze, R., Ghasem-Zadeh, A., Mbala, A. \& Seeman, E. (2013). A new method of segmentation of compact-appearing, transitional and trabecular compartments and quantification of cortical porosity from high resolution peripheral quantitative computed tomographic images. BONE, 54 (1), pp.8-20. https://doi.org/10.1016/j.bone.2013.01.007.

Publication Status:

Accepted manuscript

Persistent Link:

http://hdl.handle.net/11343/41858 